\title{
Concepts of Reactive Power Control and Voltage Stability Methods in Power System Network
}

\author{
Akwukwaegbu I. O, Okwe Gerald Ibe \\ Department of Electrical/Electronic Engineering Federal University of Technology Owerri Imo State
} (NIGERIA)

\begin{abstract}
The intent of this paper is to present an analysis of reactive power control and voltage stability in power systems. It identifies a new model used to enhance voltage stability and exposes several key issues that had remained as research challenges in this area. The steady state voltage and reactive power control in distribution systems can be properly controlled by coordinating the available voltage and reactive power control equipment, such as on-load tap-changers, substation shunt capacitors and feeder shunt capacitors. It began with an overview of reactive power and voltage stability in transmission, distribution and load, and the importance of providing reactive power locally. The description of selected control features of shunt power systems such as SVC (Static Var Compensator) - static compensators of reactive power, STATCOM-type systems (Static Compensator), static reactive power generators and systems that combine both these solutions, which are referred to as SVC based on STATCOM were not left out. It explains the need to improve the voltage stability of Power system, as well as the increasing requirements for energy quality and security. It also discusses the techniques that were adopted in controlling and monitoring of the rate of power flow in the entire power system topology. This investigates the system to an optimal level in order to reduce losses and ensures sufficiency of reactive power control during normal and emergency conditions and to prevent voltage collapse. Keywords: Reactive Power, Voltage stability, Voltage Collapse, Power system security, voltage control
\end{abstract}

\section{Introduction}

The control of voltage and reactive power is a major issue in power system operation. This is because of the topological differences between distribution and transmission systems, different strategies have evolved. This paper contains contributions of novel reactive power control and voltage stability schemes for distribution and transmission systems. A particular interest is taken to the development of control schemes to avoid so-called voltage collapse, which can result in widespread outages. In order to achieve efficient and reliable operation of power system, the control of voltage and reactive power should satisfy the following objectives [1]:

- Voltages at all terminals of all equipment in the system are within acceptable limits

- System stability is enhanced to maximize utilization of the transmission system

- The reactive power flow is minimized so as to reduce $\mathrm{R} \mathrm{I}^{2}$ and $\mathrm{X} \mathrm{I}{ }^{2}$ losses.

This ensures that the transmission system operates mainly for active power. Thus the power system supplies power to a vast number of loads and is feeding from many generating units, there is a problem of maintaining voltages within required limits. As load varies, the reactive power requirements of the transmission system vary. Since the reactive power cannot be transferred or transported over long distances, voltage control has to be effected by using special devices located through the system which possess difficulties in keeping sufficient levels of voltage in the power system network. This has been occurring practically since the first power systems started. Increasing requirements regarding both the supply reliability and quality of supplied power force using more modern (faster, more reliable, with a broader range of applications) devices. The proper selection and coordination of equipment for controlling reactive power and voltage stability are among the major challenges of power system engineering [2]. These challenges gave birth to some selected devices to control or compensate reactive power. In order to cover the additional demand for reactive power and maintain the ability to control voltage stability within the target range, various sources of reactive power, such as SVC (Static Var Compensator) - static compensators of reactive power, STATCOM-type systems (Static Compensator) static reactive power generators and systems that combine both these solutions, which are referred to as SVC based on STATCOM. In recent decades, there has been significant progress in terms of equipment designed to improve the stability of voltage in power systems. This is mainly due to the development of power supply systems in the world, which requires seeking better ways of adjusting and controlling power flows and voltage levels.

Almost all power transported or consumed in alternating current (AC) networks, supply or consume two of powers: real power and reactive power. Real power accomplishes useful work while reactive power supports the voltage that must be controlled for system reliability. Reactive power is essential to move active power through the transmission and distribution system to the customer. For AC systems, voltage and current 
pulsate at the system frequency. Although $\mathrm{AC}$ voltage and current pulsate at same frequency, they peak at different time power is the algebraic product of voltage and current. Real power is the average of power over cycle and measured by volt-amperes or watt. The portion of power with zero average value called reactive power measured in volt-amperes reactive or vars.

\subsection{Reactive Power Control in Electrical Systems}

During the daily operation, power systems may experience both over-voltage and under-voltage violations that can be overcomed by voltage/Var control [1].Through controlling the production, adsorption, and flow of reactive power at all levels in the system, voltage/Var control can maintain the voltage profile within acceptable limit and reduce the transmission losses. Transmission connected generators are generally required to support reactive power flow. For example, Transmission system generators are required by the Grid Code Requirements to supply their rated power between the limits of 0.85 power factor lagging and 0.90 power factor leading at the designated terminals. The system operator will perform switching actions to maintain a secure and economical voltage profile while maintaining a reactive power balance equation:

$\mathbf{G}-\mathbf{M}+\mathbf{S g}+\mathbf{S c}=[\mathbf{M}-\mathbf{D}+\mathbf{R}+\mathbf{S r}]$

where $\mathrm{G}=$ Generator, $\mathrm{M}=\mathrm{MVARs}, \mathrm{Sg}=$ System gain, $\mathrm{Sc}=$ Shunt Capacitor, $\mathrm{D}=$ Demand, $\mathrm{R}=$ Reactive losses, $\mathrm{Sr}=$ Shunt reactors

The 'System gain' is an important source of reactive power in the above power balance equation, which is generated by the capacitive nature of the transmission network itself. By making decisive switching actions in the early morning before the demand increases, the system gain can be maximized early on, helping to secure the system for the whole day. To balance the equation some pre-fault reactive generator use will be required. Other sources of reactive power that will also be used include shunt capacitors, shunt reactors, Static VAR Compensators and voltage control circuits

\subsubsection{Reactive power theory}

the reactive power is defined in the IEEE Standard Dictionary 100-1996 under the energy "magner" as:

$$
\text { Reactive Power }=\sum_{n=1}^{\infty} V_{n} \cdot I_{n} \cdot \sin \phi_{n} \text { ) }
$$

where $V_{n}$ and $I_{n}$ are respectively the voltage and current $r_{m s}$ values of the $n^{\text {th }}$ harmonics of the line frequency, and $I_{n}$ is the phase difference between the voltage and the current $n^{\text {th }}$ harmonics. A convention is also adopted stating that the reactive energy should be positive when the current is leading the voltage (inductive load).In an electrical system containing purely sinusoidal voltage and current waveforms at a fixed frequency, the measurement of reactive power is easy and can be accomplished using several methods without errors. However, in the presence of non-sinusoidal waveforms, the energy contained in the harmonics causes measurement errors. According to the Fourier theorem any periodic waveform can be written as a sum of sin and cosine waves. As energy meters deal with periodic signals at the line frequency both current and voltage inputs of a single phase meter can be described by -

$V(t)=\sum_{n=1}^{m} V_{n} \cdot \sqrt{2} \cdot \sin \left(n \omega_{0} t\right) \ldots m m m m m m m m m m m m m(3)$
$I(t)=\sum_{n=1}^{m} V_{n} \cdot \sqrt{2} \cdot \sin \left(n \omega_{0} t+\emptyset_{n}\right) \ldots m m m m m m m m m m m m(4)$

Where $V_{n}$ and $I_{n}$ are defined as in Equation 2.

the average active power is defined as:

Average active power $=\sum_{n=1}^{m \infty} V_{n}, I_{n} \cos \left(\phi_{n}\right) \ldots \ldots \ldots \ldots \ldots \ldots \ldots$ (5)

The implementation of the active power measurement is relatively easy and is done accurately in most energy meters in the field. 
The apparent power is the maximum real power that can be delivered to a load. As $\mathrm{V}_{\mathrm{rms}}$ and $\mathrm{I}_{\mathrm{rms}}$ are the effective voltage and current delivered to the load,

Apparent power $=\mathbf{V}_{\mathbf{r m s}} \bullet \mathbf{I}_{\mathbf{r m s}}$

The correct implementation of the apparent energy measurement is bound by the accuracy of the rms measurements.

\subsection{Importance of Reactive Power}

Voltage control in an electrical power system is important for proper operation for electrical power equipment to prevent damage such as overheating of generators and motors, to reduce transmission losses and to maintain the ability of the system to withstand and prevent voltage collapse. In general terms, decreasing reactive power causing voltage to fall while increasing it causing voltage to rise. A voltage collapse occurs when the system try to serve much more load than the voltage can support. When reactive power supply lower voltage, as voltage drops current must increase to maintain power supplied, causing system to consume more reactive power and the voltage drops further. If the current increases too much, transmission lines go off line, overloading other lines and potentially causing cascading failures. If the voltage drops too low, some generators will disconnect automatically to protect themselves. Voltage collapse occurs when an increase in load or less generation or transmission facilities causes dropping voltage, which causes a further reduction in reactive power from capacitor and line charging, and still there further voltage reductions. If voltage reduction continues, these will cause additional elements to trip, leading further reduction in voltage and loss of the load. The result in these entire progressive and uncontrollable declines in voltage is that the system unable to provide the reactive power required supplying the reactive power demands.

\subsection{Problems of Reactive Power}

Though reactive power is needed to run many electrical devices, it can cause harmful effects on your appliances and other motorized loads, as well as your electrical infrastructure. Since the current flowing through your electrical system is higher than that necessary to do the required work, excess power dissipates in the form of heat as the reactive current flows through resistive components like wires, switches and transformers. Keep in mind that whenever energy is expended, you pay. It makes no difference whether the energy is expended in the form of heat or useful work. We can determine how much reactive power your electrical devices use by measuring their power factor, the ratio between real power and true power. A power factor of 1 (i.e. 100\%) ideally means that all electrical power is applied towards real work. Homes typically have overall power factors in the range of $70 \%$ to $85 \%$, depending upon which appliances may be running. Newer homes with the latest in energy efficient appliances can have an overall power factor in the nineties. The typical residential power meter only reads real power, i.e. what you would have with a power factor of $100 \%$. While most electric companies do not charge residences directly for reactive power, it's a common misconception to say that reactive power correction has no economic benefit. To begin with, electric companies correct for power factor around industrial complexes, or they will request the offending customer to do so at his expense, or they will charge more for reactive power. Clearly electric companies benefit from power factor correction, since transmission lines carrying the additional (reactive) current to heavily industrialized areas costs them money.

\subsection{The aim of power factor correction}

Many people overlook the benefits that power factor correction can offer the typical home in comparison to the savings and other benefits that businesses with large inductive loads can expect. Most importantly, you pay for reactive power in the form of energy losses created by the reactive current flowing in your home. These losses are in the form of heat and cannot be returned to the grid. Hence you pay. The fewer kilowatts expended in the home, whether from heat dissipation or not, the lower the electric bill. Since power factor correction reduces the energy losses, you save. As stated earlier, electric companies correct for power factor around industrial complexes, or they will request the offending customer to do so, or they will charge for reactive power. They're not worried about residential service because the impact on their distribution grid is not as severe as in heavily industrialized areas. However, it is true that power factor correction assists the electric company by reducing demand for electricity, thereby allowing them to satisfy service needs elsewhere. But who cares? Power factor correction lowers your electric bill by reducing the number of kilowatts expended, and without it your electric bill will be very exorbitant.

In the most cases, PFC is used for economic reasons. Using compensating device, one can save on electricity bill as well as keep certain grid parameters determined by the energy provider. Power factor correction gives even more profits, than only savings. Compensating "unnecessary" reactive power the current carrying capacity of an existing network can be sufficient to send more active power through it, maintaining the same ratings of the apparatus within the supplying and distribution system. PFC also allows to decrease 
transmission losses and limits voltage drops. Generally, reliability of the network gets better. But one should be aware, that compensating device connected to the mains can also have negative consequences like:

- Transient generation

- Higher order harmonics generation

- Long lasting voltage rise

- Gain of higher order harmonics

- Voltage drops, outages and Overvoltage of short duration

- Other kind of distortions

\subsection{Overview on Different Voltage and Reactive Power Control Methods}

Voltage and reactive power control involves proper coordination among the voltage and reactive power control equipment in the distribution system to obtain an optimum voltage profile and optimum reactive power flows in the system according to the objective function and operating constraints. Many DNOs operate OLTC and shunt capacitors locally by using conventional controllers, e.g., voltage controller for the OLTC and either voltage, reactive power or time controllers for the capacitors; to perform basic voltage and reactive power control functions, e.g., to maintain the voltages in the distribution system within the acceptable range and to minimize power losses. Different voltage and reactive power control methods have been proposed. Properly locating and sizing shunt capacitors will decrease power losses. As an improvement to the capacitor planning based on the load size, methods to include customer load profiles and characteristics in the capacitor planning are proposed in [3]-[4]. Proper capacitor planning will also improve the voltage profile in the distribution system. The capacitor locating and sizing is studied and executed in the planning stage of the distribution system. In order to enhance the distribution system further, the capacitor should also be switched properly in the operation stage of the distribution system [4], using different types of available capacitor control. Most recently, many researchers have addressed the problem of voltage and reactive power control in distribution systems by focusing on automated distribution systems, such as in [5]-[6]. At the moment, the voltage and reactive power control based on automated distribution systems can be divided into two categories: off-line setting control and real time control [7]. The off-line setting control, for instance, aims to find a dispatch schedule for the capacitor switching and the OLTC movement based on a one day ahead load forecast. Meanwhile, the real time control, [8] for instance, aims to control the capacitor and OLTC based on real time measurements and experiences.

The application of dispatch schedule based load forecasting is motivated by the fact that although there is a random fluctuation in the load variation, the major component of the load variations is related to weather conditions. Furthermore, there is a deterministic load pattern during the day due to social activities [9]. Therefore, the load profile is quite predictable. It can be forecasted one-day-ahead with an average error less than $2 \%$ [10]. Different objective functions and operating constraints have been proposed in voltage and reactive power control with automated distribution systems. Nevertheless, all researchers [11] still consider loss Minimization and keeping the voltage within the acceptable range as the main objective and constraint in the voltage and reactive power control. Another objective that is commonly proposed is flattering the voltage profile. Commonly added operating constraints include the maximum number of OLTC operations and capacitor switchings [12], and the minimum distribution system of [13]. Other references, such as , consider minimization of OLTC operations and capacitor switching as the objective function. The automated control with off-line setting proposed in [14] fully replaces the local control operation of the conventional OLTC and capacitor operations with a remotely controlled operation. The main obstacle application of this method is its dependency on communication links and remote control to all capacitors. However, many DNOs do not have communication links downstream to the feeder capacitor locations

\subsubsection{Distribution System Voltage Control}

Distribution system voltage control is sometimes referred to as volt/var control (VVC), which Grainger and Civanlar (1985) specify as follows: The objective is to minimize the peak power and energy losses while keeping the voltage within specified limits for a variety of nominal load patterns. This objective is formulated as an optimization problem that is solved off-line, based on nominal load patterns. The optimization variables are the locations, sizes and control dead bands of capacitors and tap changer voltage regulators. Tap changers are normally automatically controlled by a relay controller that measures and regulates the secondary side voltage of the transformer. The control of transformers operating in parallel in the same substation must be coordinated to minimize circulating reactive power flows (Lakervi and Holmes, 1989). 


\subsubsection{Transmission System Voltage Control}

In practical operation of transmission systems, the voltage needs to be continuously monitored and controlled to compensate for the daily changes in load, generation and network structure. In fact, the control of voltage is a major issue in power system operation. Kundur (1994).It identifies the main objectives of voltage control as: Voltage at the terminals of all equipment in the system should be kept within acceptable limits, to avoid malfunction of and damage to the equipment. Keeping voltages close to the values for which stabilizing controls are designed, to enhance system stability and allow maximal utilization of the transmission system. Minimize reactive power flows, to reduce active as well as reactive power losses. Whereas distribution systems as a rule are operated in radial configuration. Consequently, more sophisticated control schemes than those used in distribution systems are necessary. The control of voltage is often divided into the normal, preventive and emergency state control. A brief overview of the strategies used in the different operating states follows:-

\section{(a) Primary Control}

Primary voltage controllers are used in all power systems to keep the terminal voltages of the generators close to reference values given by the operator or generated by a secondary controller. An automatic voltage regulator (AVR) acts on the exciter of a synchronous machine, which supplies the field voltage and consequently the current in the field winding of the machine and can thereby regulate its terminal voltage. The response time of the primary controller is short, typically fractions of a second for generators with modern excitation systems. Furthermore, many generators use a so-called power system stabilizer (PSS) to modulate the terminal voltage of the machine based on a local frequency measurement to contribute to damping of electromechanical oscillations. Although the power system stabilizer in most cases is integrated in the AVR, it only introduces fast oscillations around the mean value given by the AVR as long as the generator remains synchronized with the rest of the network.

\section{(b) Secondary Control}

Secondary voltage control acts on a time-scale of seconds to a minute and within regions of the network. The aim of secondary voltage control is to keep an appropriate voltage profile in a region of the system and to minimize circulating reactive power flows and maximize reactive reserves. Normally, the network is divided in a number of geographic regions. One or a few so-called pilot nodes, which are assumed to be representative of the voltage situation in the region, are selected for voltage regulation by the secondary controller. The main actuators are the set point voltages of the primary controllers of the generators within a region, although the French implementation also uses static compensation devices such as capacitor banks and reactors. The set point values are calculated by an optimization procedure using a linearized static network model to make each generator in the region contribute to the control of the pilot node voltage(s).

\section{(c) Tertiary Control}

Tertiary voltage control acts system-wide on a time scale of about ten to thirty minutes. The traditional method of tertiary control is so-called reactive power optimal power flow (OPF) (Carpentier, 1962, Dommel and Tinney, 1968). The desired operating conditions are specified in the form of a cost function, which is minimized using nonlinear optimization techniques. Usually, the main goal is to minimize losses and to keep voltages close to rated values. A secondary goal may be to maximize and distribute reactive reserves. The main control variables are voltage set points for the generators, or pilot nodes if secondary control is used, and switching orders to compensation devices such as shunt capacitors and reactors. The power flow equations (Glover and Sarma, 1994) are specified as equality constraints in the optimization whereas operational limits such as transfer limits, limits on reactive reserves and voltages are specified as inequality constraints.

\subsection{Preventive And Emergency State Voltage Control}

The operation of power systems can be divided into the states normal, alert or emergency (Kundur, 1994). The system normally operates in the normal state, but enters the alert state if the system cannot be expected to be robust to the contingencies that have been considered in the design of the system. Such situations may occur for example due to unexpected load increase or outage of some component, such that a single contingency may force the system into the emergency state. The system enters the emergency state if a severe enough contingency occurs, so that the system will experience instability or exceeds operational limits unless emergency control actions are taken. However, the system is still synchronized in the emergency state.

\subsection{Elements of the System, that Produces and absorbs Reactive Power}

Loads- a typical load bus supplied by a power system is composed of a large number of devices. The composition changes depending on the day, season and weather conditions. The composite characteristics are normally such that a load bus absorbs reactive power. Both active and reactive powers of the composite loads 
vary due to voltage magnitudes. Loads at low-lagging power factors cause excessive voltage drops in the transmission network. Industrial consumers are charged for reactive power and this convinces them to improve the load power factor.

Underground cables- they are always loaded below their natural loads, and hence generate reactive power under all operating conditions

Overhead lines- depending on the load current either absorb or supply reactive power. At loads below the natural load, the lines produce net reactive power; on the contrary, at loads above natural load lines absorb reactive power.

\subsection{Ways of Improving Voltage Stability And Control}

Reactive power compensation is often most effective way to improve both power transfer capability and voltage stability. The control of voltage levels is accomplished by controlling the production, absorption and flow of reactive power. The generating units provide the basic means of voltage control, because the automatic voltage regulators control field excitation to maintain scheduled voltage level at the terminals of the generators. To control voltage throughout the system we have to use addition devices to compensate reactive power . Reactive compensation can be divided into series and shunt compensation. It can be also divided into active and passive compensation. But mostly consideration will be focused on shunt capacitor banks, static var compensator (SVC) and Static Synchronous Compensators (STATCOM), which are the part of group of active compensators called Flexible AC Transmission Systems (FACTS). The devices used for these purposes may be classified as follows:

- Shunt capacitors

- Series capacitors

- Shunt reactors

- Synchronous condensers

- SVC

- STATCOM

\subsubsection{Shunt Capacitors}

Shunt capacitors and reactors and series capacitors provide passive compensation. They are either permanently connected to the transmission and distribution system or switched. They contribute to voltage control by modifying the network characteristics. Synchronous condensers, SVC and STATCOM provide active compensation [15]. The voltages of the buses to which they are connected. Together with the generating units, they establish voltages at specific points in the system. Voltages at other locations in the system are determined by active and reactive power flows through various elements, including the passive compensating devices [16]. The primary purposes of transmission system shunt compensation near load areas are voltage control and load stabilization. Mechanically switched shunt capacitor banks are installed at major substations in load areas for producing reactive power and keeping voltage within required limits. For voltage stability shunt capacitor banks are very useful in allowing nearby generators to operate near unity power factor. This maximizes fast acting reactive reserve. Compared to SVCs, mechanically switched capacitor banks have the advantage of much lower cost. Switching speeds can be quite fast. Current limiting reactors are used to minimize switching transients. There are several disadvantages to mechanically switched capacitors. For voltage emergencies the shortcoming of shunt capacitor banks is that the reactive power output drops with the voltage squared. For transient voltage instability the switching may not be fast enough to prevent induction motor stalling. Precise and rapid control of voltage is not possible. Like inductors, capacitor banks are discrete devices, but they are often configured with several steps to provide a limited amount of variable control. If voltage collapse results in a system, the stable parts of the system may experience damaging over voltages immediately following separation. Shunt capacitors banks are always connected to the bus rather than to the line. They are connected either directly to the high voltage bus or to the tertiary winding of the main transformer. Shunt capacitor banks are breaker-switched either automatically by a voltage relays or manually [17]. Figure 2.1 shows example of capacitor bank.

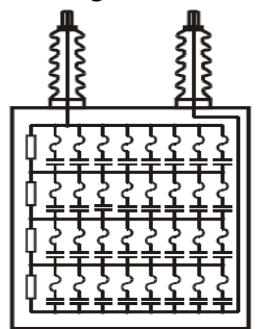

Figure 2.1 Typical capacitor bank 
The primary purpose of transmission system shunt compensation near load areas is voltage control and load stabilization. In other words, shunt capacitors are used to compensate for X I ${ }^{2}$ losses in transmission system and to ensure satisfactory voltage levels during heavy load conditions. Shunt capacitors are used in power system for power factor correction. The objective of power factor correction is to provide reactive power close to point where it is being consumed, rather than supply it from remote sources [18]. Figure 2.2 shows the influence of shunt compensation on load bus.

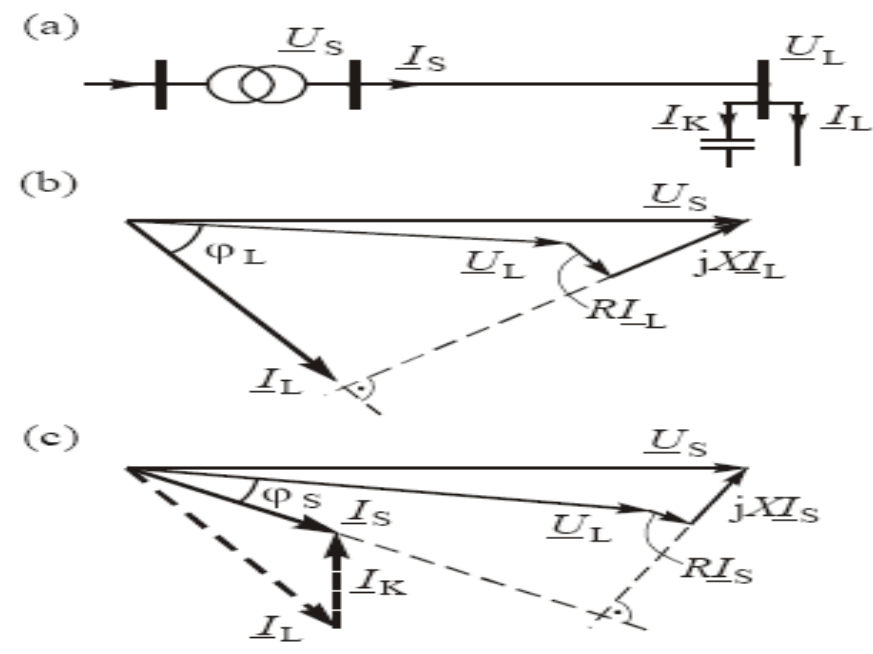

Figure 2.2 a) shunt compensation b) phasor diagram without compensationc) phasor diagram with compensation

Switched shunt capacitors are also used for feeder voltage control. They are installed at appropriate location along the length of the feeder to ensure that voltages at all points remain the allowable minimum or maximum limits as the loads vary. For voltage stability, shunt capacitor banks are very useful on allowing nearby generators to operate near unity power factor. This maximizes fast acting reactive reserve . The biggest disadvantage of shunt capacitors is that the reactive power output drops with the voltage squared. Thus, during the severe voltage decays these devices are not efficient enough . Compared to static var compensators, mechanically switched capacitor banks have the advantage of much lower cost. Switching speeds can be quite fast. Following a transmission line outage, capacitor bank energization should be delayed to allow time for line reclosing. However, capacitor switching should be before significant amounts of load are restored by transformer tap changers or distribution voltage regulators. Despite of many advantages of mechanically switched capacitors, there is couple of disadvantages as well. Firstly, for transient voltage instability, the switching may not be fast enough to prevent induction motor stalling. If voltage collapse results in system breakdown, the stable parts of the system may experience damaging over voltages immediately following separation. Over voltages would be aggravated by energizing of shunt capacitors during the period of voltage decay [2].

\subsubsection{Shunt reactors}

Shunt reactors are mainly used to keep the voltage down, by absorbing the reactive power, in the case of light load and load rejection, and to compensate the capacitive load of the line. Other equipment can be involved in the provision of reactive power and energy, such as:

- Unified Power Flow Controllers (UPFC) and other advanced FACTS (flexible ac transmission system) devices;

- Tap staggering of transformers connected in parallel;

- Disconnection of transmission lines;

- Load shedding

\subsubsection{Synchronous condensers}

Every synchronous machine (motor or generator) has the reactive power capabilities the same as synchronous generators. Synchronous machines that are designed exclusively to provide reactive support are called synchronous condensers. Synchronous condensers have all of the response speed and controllability advantages of generators without the need to construct the rest of the power plant (e.g., fuel-handling equipment and boilers). Because they are rotating machines with moving parts and auxiliary systems, they require significantly more maintenance than static compensators. They also consume real power equal to about $3 \%$ of the machine's reactive-power rating. Synchronous condensers are used in transmission systems: at the receiving end 
of long transmissions, in important substations and in conjunction with HVDC converter stations. Small synchronous condensers have also been used in high-power industrial networks to increase the short circuit power. The reactive power output is continuously controllable. The response time with closed-loop voltage control is from a few seconds and up, depending on different factors. In recent years the synchronous condensers have been practically ruled out by the. Thyristor controlled static VAR compensators, because those are much more cheaper and have regulating characteristics similar to synchronous condensers.

\subsubsection{Static VAR compensators}

An SVC combines conventional capacitors and inductors with fast switching capability. Switching takes place in the sub cycle time frame (i.e. in less than 1/50 of a second), providing a continuous range of control. The range can be designed to span from absorbing to generating reactive power. Advantages include fast, precise regulation of voltage and unrestricted, largely transient-free, capacitor bank switching. Voltage is regulated according to a slope (droop) characteristic. Static VAR compensator could be made up from:

TCR (thyristor controlled reactor);

TSC (thyristor switched capacitor);

TSR (thyristor switched reactor);

FC (fixed capacitor);

Because SVCs use capacitors they suffer from the same degradation in reactive capability as voltage drops. They also do not have the short-term overload capability of generators and synchronous condensers. SVC applications usually require harmonic filters to reduce the amount of harmonics injected into the power system by the thyristor switching. SVCs provide direct control of voltage (C.W. Taylor, 1994); this is very valuable when there is little generation in the load area. The remaining capacitive capability of an SVC is a good indication of proximity to voltage instability. SVCs provide rapid control of temporary over voltages. But on the other hand SVCs have limited overload capability, because SVC is a Capacitor bank at its boost limit. The critical or collapse voltage becomes the SVC regulated voltage and instability usually occurs once an SVC reaches its boost limit. SVCs are expensive; shunt capacitor banks should first be used to allow unity power factor operation of nearby generators.

\subsubsection{Static synchronous compensator (STATCOM)}

The STATCOM is a solid-state shunt device that generates or absorbs reactive power and is one member of a family of devices known as flexible AC transmission system (FACTS) devices. The STATCOM is similar to the SVC in response speed, control capabilities, and the use of power electronics. Rather than using conventional capacitors and inductors combined with thyristors, the STATCOM uses self-commutated power electronics to synthesize the reactive power output. Consequently, output capability is generally symmetric, providing as much capability for production as absorption. The solid-state nature of the STATCOM means that, similar to the SVC, the controls can be designed to provide very fast and effective voltage control (B. Kirby, 1997). While not having the short-term overload capability of generators and synchronous condensers, STATCOM capacity does not suffer as seriously as SVCs and capacitors do from degraded voltage. STATCOMs are current limited so their MVAR capability responds linearly to voltage as opposed to the voltage-squared relationship of SVCs and capacitors. This attribute greatly increases the usefulness of STATCOMs in preventing voltage collapse.

\subsubsection{Series capacitors and reactors}

Series capacitors compensation is usually applied for long transmission lines and transient stability improvement. Series compensation reduces net transmission line inductive reactance. The reactive generation $\mathrm{I}^{2}$ $\mathrm{X}$ compensates for the reactive consumption $I^{2} \mathrm{X}$ of the $C$ transmission line. Series capacitor reactive generation increases with the current squared, thus generating reactive power when it is most needed. This is a selfregulating nature of series capacitors. At light loads series capacitors have little effect.

\subsection{Power Capacitor Based Compensating Devices}

Power factor correction method based on power capacitors is the biggest group of the devices used in the industry and by the private users, mainly from economic reasons. On the hand, they may be a reason of unwanted distortions at the spot of operation. That is why they should be carefully selected, in accordance with actual standards. In terms of rated voltage of capacitors we can distinguish two groups of capacitor banks: 


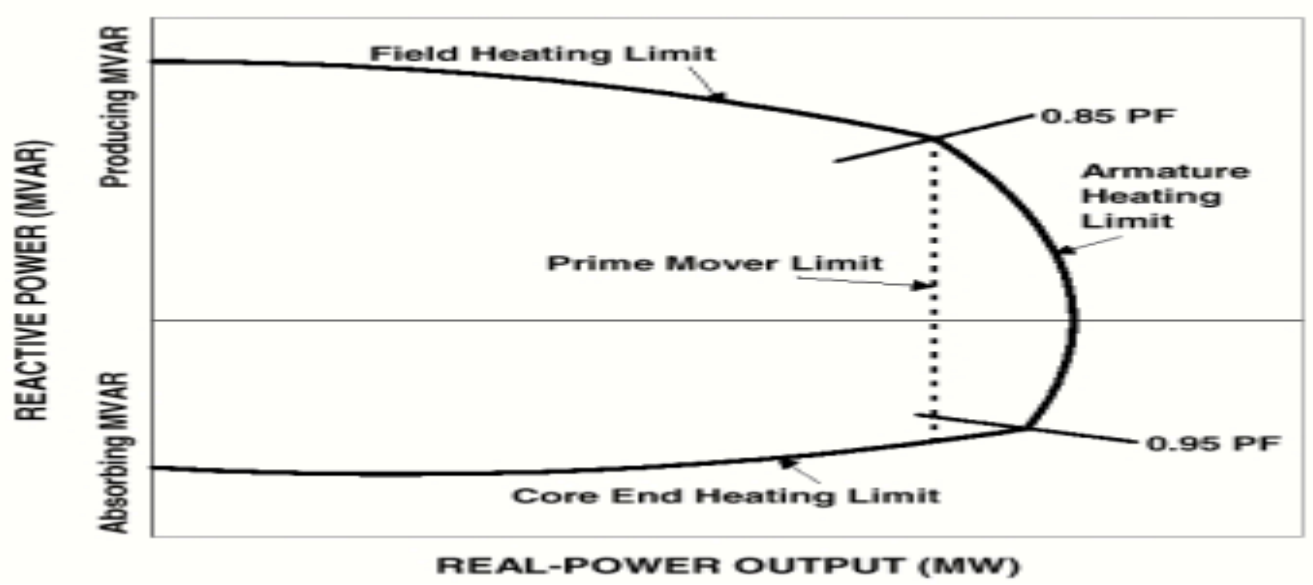

Figure 2.3. Reactive power capability dependence on real power production for a synchronous generator

Like most electric equipment, generators are limited by their current-carrying capability. Reactive power production is depended on the field heating limit and absorption on the core end-heating limit of the generator. Active power output limit is limited by armature heating. Control over the reactive output and the terminal voltage of the generator is provided by adjusting the DC current in the generator's rotating field. Control can be automatic, continuous, and fast. The inherent characteristics of the generator help maintain system voltage. At any given field setting, the generator has a specific terminal voltage it is attempting to hold. If the system voltage declines, the generator will inject reactive power in to the power system, tending to raise system voltage. If the system voltage rises, the reactive output of the generator will drop, and ultimately reactive power will flow into the generator, tending to lower system voltage. The voltage regulator will accentuate this behavior by driving the field current in the appropriate direction to obtain the desired system voltage.

\subsection{Reactive Power in Operations}

Reactive power affects power system operation in numerous ways:

1 Loads consume reactive power, so this must be provided by some source.

2 The delivery system (transmission lines and transformers) consumes reactive power, so this must be provided by some source (even if the loads do not consume reactive power). Note however that all transmission lines do provide some reactive power from their shunt line charging which offsets their consumption of reactive power in their series line losses.

3 The flow of reactive power from the supplies to the sinks causes additional heating of the lines and voltage drops in the network.

4 The generation of reactive power can limit the generation of real power. So, one primary dilemma with reactive power is that a sufficient quantity of it is needed to provide the loads and losses in the network, but having too much reactive power flowing around in the network causes excess heating and undesirable voltage drops. The normal answer to this dilemma is to provide reactive power sources exactly at the location where the reactive power is consumed. And, since strictly speaking it does not take any "fuel" to provide reactive power, it should be possible to distribute reactive power sources (such as capacitors) all around the network to avoid the problem of heating the conductors and causing voltage drops. Unfortunately, this is not practical in the extreme since there are literally millions of lines and loads connected to the grid and so this would require millions of reactive power sources - all controlled to provide exactly the right amount of reactive power at the right time every second of every day. The best we can do in most cases is work with some type of aggregation of load (say at the feeder leaving a substation) and at terminals of major lines and transformers. This also brings up the issue of the difference between power factor control (trying to exactly provide the right amount of reactive power needed to equal that which is consumed) and voltage control (trying to keep voltage levels at exactly the right level no matter how much reactive power it takes). Reactive power is both the problem and the solution to network voltage control.

\subsection{Technicals Concepts and Norms}

This paper defines the technical concepts and norms necessary for understanding the voltage instability (collapse) problem and discusses a number of related issues. Generation and absorption of active and reactive power by different power system elements were not left out. The lack of supply of reactive power in a system heavily contributes to the voltage collapse process. The relationship between reactive power and voltage stability is explained. A number of major incidents of voltage collapse have occurred in different countries in 
the past. The meaning of voltage collapse is described in simple terms and the voltage stability margin is defined. Contingencies, which are very often the cause of voltage instability, are also discussed.

The voltage collapse: This phenomenon remains a major issue for power system networks. In the words of Carson Taylor [19], "A power system at a given operating state and subject to a given disturbance undergoes voltage collapse if post-disturbance equilibrium voltages are below acceptable limits. Voltage collapse may be total (blackout) or partial. Lack of adequate reactive power resources in a power system is a major contributing factor to the process of voltage collapse. As loads in a power system increase, voltages across the network tend to decrease and reactive power losses increase as a result of high current [20]; the reactive loss is proportional to square of the current. This increased reactive power demand would be supplied by voltage regulating devices such as generators, static VAR compensators, or capacitors, if possible. However, due to physical limitations, such devices cannot supply unlimited amounts of reactive power. Often, sustained load growth will result in some source of reactive power, or perhaps a number of such sources, reaching their physical limits. Once a reactive power source has reached its maximum limit, it can no longer regulate voltage. Therefore, sustained load growth results in an accelerated voltage decay, and hence an even greater reactive power requirement. This may force other voltage regulating devices to their limits, with a subsequent further acceleration in the rate of decline of voltages. This leads to loss of voltage in a significant part of the system and is described as the voltage collapse phenomenon. The Institution of Electrical and Electronic Engineers, USA, (IEEE) [21] defines voltage collapse and voltage instability as:"Voltage collapse is the process by which voltage instability leads to loss of voltage in a significant part of the system. A system enters a state of voltage instability when a disturbance, increase in load, or system change causes voltage to drop quickly or drift downward, and operators and automatic system control fail to halt the decay. The voltage decay may take just a few seconds but can also be prolonged of the order of 10-20 minutes. If the decay continues unabated, steady state angular instability or voltage collapse will occur."Voltage collapse: can also be defined the process by which the sequence of events accompanying voltage instability leads to a low unacceptable voltage profile in a significant portion of the power system."Similarly, "Voltage stability is the ability of a power system to maintain steady acceptable voltages at all buses in the system under normal operating conditions and after being subjected to a disturbance. A system enters a state of voltage instability when a disturbance, increase in load demand, or change in system condition causes a progressive and uncontrollable drop in voltage. "The main factor causing instability is the inability of the power system to meet the demand for reactive power. The heart of the problem is usually the voltage drop that occurs when active power and reactive power flow through the inductive reactance associated with the transmission network. Voltage Instability is the absence of Voltage stability, and results in progressive voltage decrease or increase. Power System Security This is the robustness of the system to imminent disturbance and hence depends on the system operating condition as well as on the contingent probability of disturbances. Power System Stability may be broadly defined as the property of a power system that enables it to remain in a state of operating equilibrium after being subjected to a disturbance.

\section{Conclusion}

In this paper, several representative techniques of reactive power and voltage/Var control are reviewed. Their advantages and disadvantages are analyzed. The future challenge is how to efficiently and accurately solve the problem taking into consideration the dynamic nature of power systems. In its entirety, it detailed background information on the on several fundamental properties of reactive power and the consequences of shortages in reactive power reserves. Open research issues associated with voltage control and reactive power support have also been discussed. These issues are especially difficult because of the complex interaction between real and reactive power, from an engineering point of view, and from and economic point of view. It also explained the fundamentals issues on Reactive power dispatch in electric power system, as a means of injection of reactive power into the system on the generators for improving voltage stability condition when the system in heavily loaded situation.

\section{References}

[I] Proceedings of Bulk Power System Voltage Phenomena- Voltage Stability and Security. Potosi, MO, September 19-24, 1988.

[2] Proceedings of NSF Workshop on Bulk Power System Voltage Phenomena-Voltage Stability and Security. Deep Creek Lake, MD, August 4-7, 1991.

[3] Proceedings of Bulk Power System Voltage Phenomena 111- Seminar on Voltage Stability, Security and Control. Davos, Switzerland, August 22-26, 1994

[4] Proceedings of the Symposium on Bulk Power System Dynamics and Control IV-Restructuring. Santorini, Greece, August 24-28, 1998.

[5] Proceedings of Bulk Power Systems Dynamics and Control Security and Reliability in a Changing Environment. Onomichi, Japan, August 26-31, 2001.

[6] C.D. Vournas, P.W. Sauer, and M.A. Pai. Relationships between Voltage and Angel Stability of Power Systems. International Journal of Electrical Power and Energy Systems, 18:493-500, 1996.

[7] H.P. St. Clair. Practical Concepts in Capability and Performance of Transmission Lines. AIEE Dansactions, 72: 1152-1 157, 1953.

[8] C.W. Taylor. Power System Voltage Stability. McGraw-Hill, New York, 1994. 
[9] P. Kundur. Power System Stability and Control. McGraw Hill, New York, 1994.

[10] W.R. Lachs. Voltage Collapse in EHV Power Systems. Paper A78057-2, 1978 IEEE PES Winter Meeting, New York, January 28February 3, 1978.

[11] Erche, M., Petersson, T. "Reactive Power Sources". Task Force No 3. CIGRE WG 38-01. April 1987."Exchange of services between large electricity generating plants and high voltage electric power systems". Joint Working Group 39/11. CIGRE, April 1999.

[12] Gil, J.B., San Roman, T.G., Rios, J.J., Martin, P.S. "Reactive Power Pricing: a Conceptual Framework for Remuneration and Charging Procedures". IEEE Transactions on Power Systems, Vol. 15, No.2, May 2000.

[13] Hao, S.H., Papalexopoulos, A. "Reactive Power Pricing and Management ". IEEE Transactions on Power Systems, Vol. 12, No.1, February 1997.

[14] Kirby, B., Hirst, E. “Ancillary Service Details: Voltage Control”. December 1997.

[15] Larsson, M. (2000) Coordinated Voltage Control in Electric Power Systems. Doctoral dissertation, Department of Industrial Electrical Engineering and Automation, Lund University.

[16] Miller, T.J. (Editor) "Reactive Power Control in Electric Systems". John Wiley \& Sons, New York, 1982."Reactive Power: Basics, Problems and Solutions". IEEE Tutorial course. 1987

[17] Taylor, C.W. "Power System Voltage Stability". McGraw-Hill. ISBN 0-07-063184-0.1994.

[18] Trehan, N. K. "Ancillary Services - Reactive and Voltage Control”. IEEE Winter Meeting 2001. "UCTE-Principles of Network Operation”. February 1999. Available from www.ucte.org

[19] Xu, W., Zhang, Y., da Silva, L., Kundur, P. "Competitive Procurement of Dynamic Reactive Power Support Service for Transmission Access". IEEE Summer Power Meeting 2000. Whitehead, A. “A New Market in Reactive Power". Birmingham, UK. April1998.

[20] IEA, Renewable Energy, Market and Policy Trends in IEA Countries, International Energy Agency, France, 2004.

[21] ESHA, "European Renewable Energy Review 2006", European Small Hydropower Association, 2007. http://www.esha.be/fileadmin/ esha_files/documents/publications/articles/ERER2006-Final.pdf, last accessed on 22 Sept, 2007. 